It now becomes necessary to show that the normal series $E$, $E_{1}, E_{2}, \cdots ; E, E_{1}, F_{1}, F_{2}, \cdots$ define the same complementary series, and similarly for $E, E_{1}^{\prime}, E_{2}^{\prime}, \cdots ; E, E_{1}^{\prime}, F_{1}, F_{2}, \cdots$. This amounts to proving the theorem for the algebras $E_{1}$ and $E_{1}^{\prime}$, which involves a finite number of repetitions of the above proof.

$A$ chief or principal series $E, P_{1}, P_{2}, \ldots$ of an algebra being defined as one in which $P$ is the maximal subalgebra of $P_{s-1}$ which is invariant is $E\left(P_{0}=E\right)$ it is easily shown in the symbolic notation, by exactly the same process as for the normal series, that the system of complementary algebras $C_{1}, C_{2}, \cdots$ is independent of the chief series selected.

In the case of the normal series the complementary algebras $K_{1}, K_{2}, \cdots$ are necessarily simple, but this is not true of the complementary algebras $C_{1}, C_{2}, \ldots$ in the case of the chief series.

The University of Colorado, BOULDER, COLO.

\title{
A GEOMETRIC PROPERTY OF THE TRAJECTORIES OF DYNAMICS.
}

BY DR. EDWARD KASNER.

(Read before the American Mathematical Society, September 7, 1905.)

SuPPOSE that the force acting on a particle whose coordinates are $x, y, z$, produces an acceleration having the components $\phi(x, y, z), \psi(x, y, z), \chi(x, y, z)$. The equations of motion are then

$$
\ddot{x}=\phi(x, y, z), \ddot{y}=\psi(x, y, z), \ddot{z}=\chi(x, y, z),
$$

where dots denote differentiation with respect to the time $t$. In such a field of force the initial position and initial velocity completely determine the trajectory. The totality of trajectories thus constitutes a quintuply* infinite system of space curves.

Consider now those trajectories obtained by starting the par-

* The only exception arises in the trivial case where the force is everywhere zero. Then the trajectories are the fourfold infinity of straight lines. 
ticle at a given point $P$ in a given direction. These form a simply infinite system since the initial speed $v$ is arbitrary.

The osculating plane at $P$ must contain both the given direction and the direction of the force acting at $P$; hence the $\infty^{\prime}$ trajectories have the same osculating plane. The osculating sphere, however, varies from trajectory to trajectory. We now prove that, no matter what the law of force, the locus of centers of these $\infty^{1}$ osculating spheres is a straight line.

The formulas for the center of the osculating sphere of any space curve are $\dagger$

$$
\begin{aligned}
& X=x+r^{2} x^{\prime \prime}-\operatorname{\rho rr} r^{\prime}\left(y^{\prime} z^{\prime \prime}-z^{\prime} y^{\prime \prime}\right), \\
& Y=y+r^{2} y^{\prime \prime}-\operatorname{\rho r} r^{\prime}\left(z^{\prime} x^{\prime \prime}-x^{\prime} z^{\prime \prime}\right), \\
& Z=z+r^{2} z^{\prime \prime}-\operatorname{\rho rr}\left(x^{\prime} y^{\prime \prime}-y^{\prime} x^{\prime \prime}\right),
\end{aligned}
$$

where accents denote differentiation with respect to the arc $s$; $r$ is the radius of curvature, and $\rho$ the radius of torsion. These are determined by the familiar formulas

$$
\begin{gathered}
1 / r^{2}=x^{\prime \prime 2}+y^{\prime \prime 2}+z^{\prime 2}=\Sigma x^{\prime 2}, \\
\frac{1}{\rho}=-\frac{1}{\Sigma x^{\prime \prime 2}}\left|\begin{array}{ccc}
x^{\prime} & x^{\prime \prime} & x^{\prime \prime \prime} \\
y^{\prime} & y^{\prime \prime} & y^{\prime \prime \prime} \\
z^{\prime} & z^{\prime \prime} & z^{\prime \prime \prime}
\end{array}\right| .
\end{gathered}
$$

Differentiating (3), we find

$$
r^{\prime}=-r^{3} \Sigma x^{\prime \prime} x^{\prime \prime \prime}
$$

To apply these formulas to the trajectories of dynamics, it is necessary to replace arc derivatives by time derivatives. The transformation formulas are

$$
\begin{aligned}
x^{\prime} & =\dot{x} / v, \\
x^{\prime \prime} & =(v \ddot{x}-\dot{v} \dot{x}) / v^{3}, \\
x^{\prime \prime \prime} & =[v(\ddot{v}-\ddot{v} \dot{x})-3 \dot{v}(v \ddot{x}-\dot{v} \dot{x})] / v^{5},
\end{aligned}
$$

with similar results for $y$ and $z$.

\footnotetext{
* See Scheffers, Theorie der Curven, Leipzig (1901), p. 234.
} 
Here $v$ denotes the velocity, so that

$$
v^{2}=\Sigma \dot{x}^{2}, \quad v \dot{v}=\Sigma \dot{x} \ddot{x}, \quad v \ddot{v}+\dot{v}^{2}=\Sigma \ddot{x} \ddot{x}+\Sigma \ddot{x}^{2} .
$$

By means of formulas (2) to (7), the coördinates of the center of the osculating sphere may be expressed in terms of $x$, $y, z$ and their time derivatives of first, second and third orders.

We now simplify the discussion by means of a particular choice of axes. Let the given point $P$ be taken as origin; the given direction, as axis of $x$; and let the fixed osculating plane be the plane $x y$. Since the initial velocity is directed along the axis of $x$, we have

$$
\dot{x}, \dot{y}, \dot{z},=v, 0,0 .
$$

The force acts in the plane $x y$, hence the acceleration components are

$$
\ddot{x}, \ddot{y}, \ddot{z}=\phi, \psi, 0,
$$

where $\phi, \psi$ are constants since $x, y, z,=0,0,0$. The third derivatives are

$$
\dddot{x}, \dddot{y}, \ddot{z},=v \phi_{x}, v \psi_{x}, v \chi_{x} .
$$

From (6), we can now find the arc derivatives

$$
\begin{aligned}
x^{\prime}, y^{\prime}, z^{\prime} & =1,0,0, \\
x^{\prime \prime}, y^{\prime \prime}, z^{\prime \prime} & =0, \quad \psi / v^{2}, 0, \\
x^{\prime \prime \prime}, y^{\prime \prime \prime}, z^{\prime \prime \prime} & =\left(-\psi^{2}, v^{2} \psi_{x}-3 \phi \psi, v^{2} \chi_{x}\right) / v^{4} .
\end{aligned}
$$

Substituting these values in (3), (4), (5), we have

$$
r=\frac{v^{2}}{\psi}, \quad \rho=-\frac{\psi}{\chi_{x}}, \quad r^{\prime}=\frac{3 \phi \psi-v^{2} \psi_{x}}{\psi^{2}} .
$$

Finally, from (2), we find

$$
X=0, Y=\frac{v^{2}}{x}, Z=\frac{3 \phi \psi-v^{2} \psi_{x}}{\psi \chi}
$$

Here we note that $X, Y, Z$ are linear functions of the parameter $v^{2}$. Elimination thus gives the straight line

$$
X=0, Z=\frac{3 \phi}{\chi_{x}}-\frac{\psi_{x}}{\chi_{x}} Y
$$

as the required locus of centers. 
Incidentally, the value of $\rho$ obtained in (9) shows that the torsion, unlike the curvature, is independent of $v$.

An arbitrary field of force (1) produces $\infty^{5}$ trajectories, of which $\infty^{1}$ pass through a given point in a given direction. These $\infty^{1}$ trajectories have, at the given point, a common osculating plane and a common torsion. The locus of centers of their osculating spheres is a straight line. Thus every field of force gives rise to a correspondence between the direction elements and the straight lines of space.

Columbia University, August, 1905.

\section{ON THE POSSIBLE NUMBERS OF OPERATORS OF ORDER 2 IN A GROUP OF ORDER $2^{m}$.}

BY PROFESSOR G. A. MILLER.

(Read before the American Mathematical Society, September 7, 1905.)

It is well known that every group of order $2^{m}$ which contains only one operator of order 2 is either cyclic or it is composed of the cyclic group of order $2^{m-1}$ and $2^{m-1}$ operators of order 4 transforming each operator of this cyclic group into its inverse.* There are exactly two such groups for every value of $m>2$. When $m=3$ the latter of these two is the quaternion group, and when $m<3$ the cyclic group is the only one that contains only one operator of order 2 .

The groups of order $2^{m}$ in which the number of all the operators of order 2 is $\equiv 1$ mod. 4 have been determined incidentally in a recent paper. $\dagger$ Such groups exist only when the number of operators of order 2 is of the form $2^{k}+1$, and there are exactly two possible groups for every arbitrary value of $k$. One of these is the dihedral rotation group of order $2^{k+1}$, and the other is obtained by adding to the cyclic group of order $2^{k+1}$ an operator of order two which transforms each of its operators into its $\left(2^{k}-1\right)$ th power. Just half of the additional operators are of order two and the others are of order 4.

For instance, there are just two groups whose orders are of the form $2^{m}$ and which contain just five operators of order two;

* Burnside, Theory of groups, 1897 , p. 75.

†Transactions Amer. Math. Society, vol. 6 (1905), p. 62. 